\title{
Light, rapid rural appraisal : des méthodologies brillantes et légères?
}

\section{Abdou Salam Fall et André Lericollais}

\section{(2) OpenEdition}

\section{Journals}

Édition électronique

URL : http://journals.openedition.org/apad/376

DOI : 10.4000/apad.376

ISSN : 1950-6929

Éditeur

LIT Verlag

Édition imprimée

Date de publication : 15 mars 1992

\section{Référence électronique}

Abdou Salam Fall et André Lericollais, « Light, rapid rural appraisal : des méthodologies brillantes et légères ? », Bulletin de l'APAD [En ligne], 3 | 1992, mis en ligne le 21 juillet 2006, consulté le 07 septembre 2020. URL : http://journals.openedition.org/apad/376 ; DOI : https://doi.org/10.4000/apad. 376

Ce document a été généré automatiquement le 7 septembre 2020.

Bulletin de l'APAD 


\title{
Light, rapid rural appraisal : des méthodologies brillantes et légères?
}

\author{
Abdou Salam Fall et André Lericollais
}

1 Fortement sollicités et approuvés par les financeurs et par les développeurs, les méthodologues de la recherche en milieu rural s'efforcent de concevoir, de promouvoir et finalement d'imposer des pratiques qualitatives, rapides et interactives, pour la production d'un savoir ciblé, immédiat et opérationnel. Les réflexions du groupe AMIRA dans les années 1980 sur ce thème apparaissent très timides comparées aux méthodes de Rapid Rural Appraisal. Le RRA que l'on traduit généralement par investigation rapide en milieu rural ou plus justement par diagnostic rapide pour le développement en milieu rural, est mis en pratique et enseigné à l'instigation de chercheurs anglo-saxons aussi renommés que R. Chambers ou à l'initiative d'intervenants tel le CRDI canadien. L'approche demeure éminemment anthropologique en ce sens qu'elle se fonde sur les savoirs paysans et leurs pratiques. Mais alors que la démarche habituelle des ethnologues cherchant à saisir de l'intérieur un univers totalement étranger, supposait l'immersion et une patiente approche participative, que la production scientifique s'échelonnait dans le temps, qu'enfin les changements étaient plutôt déplorés comme autant de dégradations d'un ordre ancien supposé cohérent et harmonieux, le RRA s'intéresse aux dynamiques actuelles et aux problèmes immédiats, l'analyse est mise en forme sans délais, validée sur place et prévue pour éclairer l'action. Elle sert le cas échéant à préparer des recherches approfondies.

Le Rapid Rural Appraisal (RRA), part d'une contestation vive de pratiques de recherche supposées habituelles

2 Il considère que les pratiques de l'expertise sont déplorables, que les coûts, la lourdeur, la lenteur de la recherche conventionnelle, ses incapacités et ses insuffisances face à la demande sociale, face à l'urgence... ne sont pas inéluctables. A l'origine du RRA il y a 
l'insatisfaction des chercheurs eux-mêmes, et la remise en question de leurs propres pratiques.

3 La recherche rapide n'est évidemment pas une nouveauté. Le RRA se situe d'emblée sur le terrain d'une recherche trop souvent expédiée et sans principes, pour en dénoncer les pratiques, en stigmatisant (Chambers,1990) une recherche facile, sans méthodologie avouable et où la référence au terrain sinon à la réalité apparaît accessoire voire facultative. Seule importe la cohérence du discours, qu'il soit conformiste ou polémique.

Mais le RRA se définit aussi en réaction contre "l'enlisement" de la recherche conventionnelle, notamment la recherche fondée sur des enquêtes statistiques lourdes ou des investigations à caractère exhaustif. Effectivement, dans les archives des centres de recherches dorment des enquêtes statistiques qui ne furent que très partiellement exploitées quand elles ne se sont pas révélées inexploitables. Au niveau de recherches plus modestes, nous savons que les fastidieux relevés de temps de travaux ou les suivis budgétaires en vogue jusque dans les années 1970 n'ont souvent donné lieu qu'à des exploitations succinctes. Il aurait fallu au préalable un diagnostic englobant et rapide, avant de se lancer dans ces relevés fastidieux. Les méthodologues du RRA tentent d'organiser cette démarche avec rigueur et précision.

5 Mais qui plus est, d'une façon générale, l'enquête quantitative est vigoureusement mise en question. Le qualitatif est plus à même de découvrir les phénomènes structurants. Les protocoles lourds transforment la pratique de la recherche en tâches qui accaparent totalement le chercheur, réduisent sa liberté, absorbent son imagination, inhibent l'inspiration. Les chiffres ont trop de pouvoirs. Ce ne sont que des indications à utiliser avec réserve puisqu'ils simplifient et parfois dénaturent les faits dont ils sont censés rendre compte. La complexité ne peut être restituée que par le qualitatif. Ainsi pour le RRA il est clair que le qualitatif prime sur le quantitatif.

6 La méthodologie préconise et exige une finalisation immédiate et efficace. Elle rejette les conclusions écrites en vue de l'action par des évaluateurs expéditifs comme étant purement spéculatives et personnelles. Elle déplore la pratique des chercheurs qui après avoir recueilli longuement une information considérable mais sans objectifs de développement clairement partagés avec les enquêtés, disparaissent pendant des mois voire des années pour produire un travail académique qui restera totalement ignoré des intéressés. Le produit de RRA se fait en équipe et en interaction avec les acteurs paysans, s'élabore de manière itérative et se trouve de ce fait validé par les parties prenantes. Quelques indications chiffrées, quelques schémas de cartes ou de transects suffisent pour confirmer ou illustrer l'analyse. Un diagnostic syncrétique et éclairant est la forme requise. L'essentiel est que des réponses pertinentes soient apportées aux questions-problèmes. La compétence des chercheurs doit se traduire par des suggestions et des propositions nouvelles.

7 La recherche classique est critiquée pour ses lenteurs et ses orientations. Elle est considérée comme étant plus apte à restituer des situations historiques qu'à jouer le rôle de révélateur des changements en cours. Par conséquent elle apparaît peu opérationnelle face à l'urgence et à la demande d'information qu'exige l'action.

Sur quelles innovations méthodologiques se fonde le "RRA" ?

8 L'approche se veut documentée au départ, exploratoire, qualitative, ciblée, participative, interactive et itérative sur le terrain. Ajoutons la nécessité de la restitution comme objectif de la démarche. Tels sont les termes clés de cette 
méthodologie qui se veut innovante pour la collecte de l'information et performante en terme de développement.

9 En quoi innove-t-on par rapport aux très habituelles recherches cursives si fréquemment pratiquées par les experts, des universitaires et certains chercheurs qui n'ont ni les moyens ni le temps de s'attarder sur le terrain?

10 1. Le RRA se fait en équipe pluridisciplinaire. Les chercheurs s'impliquent tous sur le terrain. Des disciplines différentes confrontent leurs modes d'investigation et leurs résultats. Des séances de mise en commun ont lieu quotidiennement. La collecte et l'analyse des données se font simultanément. Le RRA requiert des chercheurs expérimentés, à l'esprit ouvert. L'interdisciplinarité est l'approche privilégiée. Elle signifie dialogue entre les chercheurs sur le terrain dans le sens d'une complémentarité pour une compréhension la plus étoffée et la plus pertinente des faits étudiés.

11 2. La "triangulation" est l'un des principes clés du RRA. Elle postule le recours à une diversité de sources d'information et à leur croisement afin que s'expriment les complexités à côté des singularités. Une stratification minimale est dès lors nécessaire pour une approche syncrétique. Elle est fondée sur un choix raisonné dont l'objectif est de prendre en compte les éléments qualitativement représentatifs, explicatifs ou structurant du phénomène diagnostiqué. La triangulation suggère une mise en parallèle de sources variées en fonction d'une diversité de modes, de lieux et d'outils de collecte et de traitements de l'information. Par cette volonté de diversifier, on veut éviter le glissement trop habituel vers l'exhaustivité qui est l'une des trames du perfectionnisme. Pour ce faire la triangulation est couplée avec le principe tout aussi fondamental du RRA, "l'ignorance optimale".

12 3. Le principe de "l'ignorance optimale" peut se résumer comme une approche ciblée, pertinente et négligeant tout ce qui peut paraître secondaire vis à vis de l'objet de recherche.

13 4. La philosophie du RRA est fondée sur les dispositions des chercheurs. Ceux-ci doivent se mettre à l'écoute des communautés étudiées tout en leur apportant les compétences nécessaires à l'analyse de leurs situations-problèmes. L'approche préconisée est interrogative avec le recours à des techniques et à des procédés empiriques et actifs. Les outils méthodologiques sont essentiellement exploratoires et participatifs. Les informations collectées sont principalement qualitatives.

Le RRA emploie une batterie d'outils de collecte et d'analyse de données

14 1. L'interview semi-directif est la technique la plus courante en RRA. C'est un entretien approfondi avec des informateurs-clés ou personnes-ressources de la communauté étudiée. Le chercheur dispose d'un guide d'entretien semi-structuré lui permettant d'organiser un débat libre et centré sur l'objet de recherche. L'identification des informateurs-clés ainsi que la conduite d'entretiens semi-directifs appellent une compétence et une expérience certaines du chercheur à qui il appartient par la suite de procéder à une analyse de contenu des entretiens recueillis.

15 2. Les jeux analytiques sont multiples. Trois types peuvent être relevés à titre d'exemple d'interview de groupe : le classement par ordre de préférence, le classement par niveau de prospérité et le Focus group.

- Le classement par ordre de préférence : il consiste, par un jeu simple, à établir les priorités ou préférences d'une communauté ou d'un groupe. Dans un premier temps, le groupe est invité à proposer une liste de priorités ou de préférences. Ensuite, chaque choix est inscrit 
sur une fiche séparée et facilement identifiable. Il est couplé avec un autre choix et on demande à tous les informateurs-clés, à tour de rôle, de donner leur préférence et les raisons qui la fondent. Un classement final est fait et une importante moisson d'informations est ainsi recueillie permettant une analyse des besoins et préférences de la communauté en question.

- Le classement par niveau de prospérité : il vise à identifier les critères et indicateurs de richesse et les modalités d'accès aux ressources, critères fonctionnels dans le vécu social des intéressés. Il met aussi à contribution différents informateurs-clés à qui on fait classer les membres de la communauté, grâce à des fiches individuelles, selon leur niveau de prospérité. Pour toute personne classée, les raisons sont relevées. Les différents groupes constitués permettent une stratification de la communauté. Les critères de différenciation implicites et opérationnels sont pris en compte pour une analyse des caractéristiques socio-économiques de la communauté considérée (FALL A.S.,1991).

- Le Focus group : Il s'agit d'organiser des discussions de groupe homogène de 6 à 12 personnes autour d'un thème d'intérêt commun. L'objectif recherché est de susciter une discussion libre permettant l'expression de tous et non le consensus. Les différents messages-clés font l'objet d'une analyse de contenu par l'équipe des chercheurs (SIMARD, 1989).

3. L'observation est directe. L'accent est mis sur l'œil exercé des chercheurs et les observations se font avec la communauté en question. C'est ainsi que des cartes et transects géographiques sont réalisés pour spatialiser; des diagrammes et calendriers d'activités et saisonniers établis pour illustrer et visualiser. Les chercheurs sélectionnent des indicateurs accessibles à l'observation directe.

4. Les portraits et biographies sont le moyen d'appréhender la situation et la dynamique sociales. L'approche biographique reconstitue les histoires de vie d'individus grâce à différentes sources. Les trajectoires individuelles et collectives, sont analysées dans le contexte de leurs relations sociales. La société peut s'étudier au travers de l'expérience des acteurs sociaux. Ce n'est pas seulement la vie de l'élite qui renseigne sur l'évolution sociale, l'expérience de simples gens de la société civile peut être tout aussi instructive.

5. Les données secondaires jouent un rôle important dans la démarche. L'équipe de chercheurs doit s'appuyer sur une exploitation préalable de ces données, de manière à centrer pertinemment ses investigations. Le recours à la documentation publiée ou souterraine et informelle, à l'imagerie et aux photographies aériennes disponibles, aux documents administratifs et locaux est particulièrement recommandé.

La restitution permanente donne tout son sens à la démarche

Elle procède de la logique participative de la communauté d'étude au RRA. La restitution est un moment de dialogue entre équipe de recherche et communauté étudiée. Elle permet d'affiner les résultats du diagnostic rapide et de les faire valider. La restitution est une étape interrogative qui peut occasionner une appropriation des résultats du diagnostic par la communauté ou, susciter un intérêt renouvelé pour la réflexion-bilan sur une situation-problème car il n'y a pas de connaissance là où il n'existe pas d'effort systématique de penser le réel social.

Le RRA peut être classé en plusieurs types

(McCraken J.A. et al. 1988). 
21 1. Le RRA exploratoire : L'équipe intervient dans une phase avant projet pour aider à identifier des orientations pour un travail de développement ou à formuler des hypothèses pour la recherche approfondie. Le RRA ne se substitue pas à la recherche mais la facilite.

22 2. Le RRA thématique: L'équipe de recherche se focalise sur un centre d'intérêt spécifique qu'une connaissance préalable a contribué à repérer. Elle tente de répondre qualitativement à une question-clé. La méthode a donné récemment des résultats intéressants en Casamance pour l'analyse institutionnelle d'associations paysannes inter-villageoises (Bassène M. et al., 1991).

23 3. Le RRA participatif : Ce type de RRA met en évidence les talents d'animation de groupe de l'équipe de chercheurs. Il consiste, par des procédés techniques simples d'impliquer la communauté à toutes les étapes d'identification, de mise en œuvre et d'évaluation du programme.

24 4. Le RRA de contrôle : Ce RRA concerne l'évaluation d'un programme ou l'étude d'impact d'un projet.

25 Cette classification est faite à des fins didactiques. La réalité est plus complexe car un RRA peut être, par exemple, à la fois exploratoire et participatif, comme il peut être thématique et de contrôle.

26 Ces méthodologies, dont la plupart des techniques constitutives sont connues, ont pour objectif et pour originalité de baliser et d'orienter fermement l'ensemble de la recherche, de ne rien laisser à l'improvisation hasardeuse, d'obliger le chercheur à participer à une dynamique de groupe, d'impliquer la population soumise à l'enquête afin qu'elle soit elle-même engagée par ses conclusions.

La démarche a ses limites

27 La mise en œuvre de cette nouvelle méthodologie ne va pas de soi. Beaucoup d'obstacles sont prévisibles, cette approche a ses présupposés qu'il est loisible de discuter.

Certaines limites sont immédiatement apparentes. Le travail en équipe et l'exercice de la pluridisciplinarité au même moment et au même lieu créent inévitablement des tensions qui ne sont pas dues seulement à l'individualisme irrépressible des chercheurs, mais à la difficulté qu'ils rencontrent pour mettre en œuvre correctement leurs techniques d'investigation, pour développer leur point de vue dans sa totalité avec les références de la discipline, pour accéder en temps utile et sous la forme adéquate à l'information tenue par d'autres... La confrontation dans un espace confiné devient forcément conflictuelle et requiert une autorité ou un habile médiateur, pour maintenir la communication. Le temps nécessaire pour préparer la recherche de terrain puis pour en maîtriser les matériaux n'est-il pas minimisé à l'extrême? Ajoutons que les moyens modernes de saisie et de traitement de l'information même qualitative sont ignorés ou sont passés sous silence.

Le savoir des paysans est une mine sans fond où il n'y a qu'à puiser. Ceux-ci se doivent d'être présents, savants, cohérents et toujours bien disposés. Il semble important que la participation des villageois soit sans faille et constructive dans cet exercice fastidieux. Le fond du problème est cette valorisation a priori des savoirs et de la science des paysans. L'idéologie populiste est bien l'un des fondements des RRA (Olivier de Sardan J.P., 1991). C'est une position intellectuelle pour le moins optimiste. 
30 Le débat méthodologique pour des pratiques de recherche pluridisciplinaires n'est-il pas un pseudo-débat puisque fondamentalement l'innovation a lieu au sein de la discipline qui la met en œuvre. Elle y sera jugée à ses performances et en fonction de la validité des résultats obtenus. La question de l'allégement des méthodes se pose en premier lieu au niveau de chaque discipline dont on a tendance à oublier, pour le moins, les savoirs-faire et les spécificités.

31 Peut-on tout attendre de techniques qualitatives et socio-économiques? Les restitutions faites sous l'arbre à palabre peuvent se révéler normatives et interprétatives. On retrouve le vieux débat sur la norme et la réalité. Les contradictions que l'exercice de «triangulation » risque fort de faire apparaitre seront à réduire, mais la discussion n'aboutit pas toujours à ce résultat. Enfin l'immersion sociale n'apparaît plus nécessaire pour communiquer et connaître. Il est vrai que l'expérience exigeait un engagement personnel quasi total et que le temps ne soit pas compté, vu ce qu'elle impliquait d'apprentissage de la langue, d'initiation à l'éthique pour une mise au diapason minimale. L'approche brève et en équipe limite toute immersion et peut biaiser la communication. Pourtant les avantages d'une insertion patiente réelle et assumée ne peuvent pas être mis en doute. Pour en apprécier la valeur et la richesse il suffirait de lire le dernier livre sur les Touaregs que vient d'écrire E. Bernus (Bemus E., 1991).

32 Il est facile de montrer que le quantitatif demeure tout à fait nécessaire pour vérifier, confronter, mais aussi pour découvrir (Couty Ph. et Win ter G, 1983). Tous les chiffres, tous les coefficients ne peuvent pas être mis en cause. Certains sont fort bien compris par les paysans, dès qu'il s'agit des aspects concrets de la vie rurale. On pourrait évoquer la dynamique du peuplement si essentielle et si difficile à cerner correctement sans recensement attentif. Pour l'analyse de la gestion foncière, il y a les modalités d'accès à la terre mais aussi les superficies concernées. Les deux types d'informations se complètent nécessairement. L'équipement agricole doit être dénombré avant d'en apprécier l'état et les fonctions. La gestion des cheptels peut-elle être analysée sans la connaissance des effectifs ? Les performances de l'agriculture sont affaire de décision et de pratiques mais ont une traduction évidente et éclairante en chiffres de superficies, de productions et de rendements. Tout cela peut être recensé et mesuré. Les évaluations qualitatives sur le niveau des ressources et la situation vivrière sont trop approximatives et incertaines. Les descriptions d'évolutions risquent de dramatiser sans que personne dans la population enquêtée n'ait envie de contester. Il faut bien aller voir de plus près et plus souvent, notamment quand il s'agit des questions vitales ou de faits controversés.

L'approche préconisée est par définition brève et ponctuelle. Comment étendre les conclusions dans le temps et l'espace ? La référence aux structures n'est guère explicite qu'il s'agisse des structures sociales dans leurs rapports avec l'organisation de la production, ou des structures agraires visibles dans le paysage. Leur repérage requiert des analyses classiques en sociologie ou en géographie agraire, qu'il faudra bien faire. Les problèmes de validation et de généralisation des résultats à une échelle significative ne peuvent pas être éludés.

La restitution enfin, n'a de sens que si le savoir acquis dépasse ce que les gens pratiquent, et ce que les plus avisés expriment. La simple confrontation-restitution peut tourner à la confirmation simpliste colorée de complaisance. Les informations chiffrées sur les techniques ou les pratiques en vigueur intéressent aussi les paysans. La 
présentation de résultats obtenus par la mise en œuvre d'innovations testées dans le milieu stimuleront à coup sûr le débat, autant que de simples suggestions, mais cela implique une présence longue et souvent des protocoles de recherche lourds.

La recherche doit-elle se réduire à des objectifs directement utilitaires et la production scientifique se limiter à des rapports de circonstance ? Et le public qui peut s'intéresser à ces travaux doit-il se circonscrire à la population qu'ils concernent et à ceux qui pourraient intervenir?

La recherche pour être créatrice doit demeurer constamment ouverte. Elle exige une totale liberté d'esprit et de pensée. Elle a pour tâche de dépasser les opinions conjoncturelles qui peuvent s'exprimer et les apparences immédiates que l'on peut repérer. Elle se doit de les mettre en accusation les unes et les autres par des investigations systématiques et concertées. La mise au point de la méthodologie est pour chaque programme un objet de recherche et de discussion. Elle est choisie et se module en fonction des objectifs. Légiférer en la matière risque d'être extrêmement limitatif.

Entre le RRA et les recherches approfondies plus classiques il y a complémentarité

La réflexion méthodologique autour du RRA est tout à fait salutaire pour stigmatiser les pratiques improvisées de recherches expédiées en tous genres. Elle est certainement utile pour orienter et organiser une recherche, pour rappeler certaines exigences trop souvent oubliées, mais la nécessité de conserver l'initiative, d'en passer par des investigations approfondies, par des mesures précises et parfois longues, ne peut être aussi facilement évacuée. Nous constatons d'ailleurs que parmi les méthodologies préconisées toutes ne sont pas légères et accessibles, loin s'en faut; certaines requièrent des moyens et des compétences techniques pour être mises en œuvre correctement. Il est évident que l'activité de recherche doit se développer en fonction d'objectifs clairement identifiés avec les méthodologies adaptées. Mais même dans une perspective de "recherche-développement" l'investigation rapide, qualitative et interactive ne saurait suffire dans tous les cas. Une fois passée l'équipe météorique et le nuage des questions-réponses retombé, le chercheur dit conventionnel pourra reprendre son cheminement en constatant, l'étonnement des villageois devant tant d'empressement et de sollicitations.

38 Face à la complexité des pratiques et des dynamiques paysannes, il n'est pas mauvais que le jeune chercheur éprouve le sentiment d'une irréductible opacité du réel, jusqu'à l'angoisse. Chemin faisant, il en découvrira quelques facettes, risquera des mises en ordre, esquissera des perspectives. La confrontation avec les autres disciplines sera éprouvante et déstabilisante. L'insatisfaction est inhérente au métier, l'essentiel est la progression acquise au prix d'un cheminement exigeant et rigoureux.

On aura compris que la tâche d'une équipe RRA, tentant de faire émerger et de nous restituer un "projet paysan" en quelques heures, n'est pas aisée, et qu'il n'y a pas lieu de s'en tenir à cette méthodologie un peu dogmatiquement révélée et enseignée.

La pratique du RRA est évolutive. Au fil des expériences, des résultats incontestablement intéressants sont obtenus dans une perspective d'action. Cependant en terme de recherche et de production de connaissances ce n'est pas réduire l'intérêt de la démarche que de la situer de façon privilégiée à l'amont de la recherche approfondie, pour la préparer, la faciliter et l'orienter. 


\section{BIBLIOGRAPHIE}

Ancey G., 1984, Enquêtes rurales en Afrique sur échantillons restreints. Paris, AMIRA Nº 45, 157 p.

Bassene M., Diame F., Ellsworth L. et al., 1991, Perspectives de renforcement de la FADECBA

(Fédération pour le développement communautaire de Balantacounda). Dakar, PRAAP, 91 p. multig.

Bernos E., 1991, Touaregs. Chronique de l'Asawak. Paris, Calmann-Lévy 175 p.

Chambers R., 1990, Développement rural. La pauvreté cachée. Paris Karthala / CTA, 374 p.

Chambers R., 1990, Diagnostic rapide et participatoire sur terrain rural Hyderabad, Administrative Staff College of India , 8 p.

Couty P. et Winter G., 1983, Qualitatif et quantitatif, deux modes d'investigation complémentaires. Paris, AMIRA N 43, 78 p.

Fall A.S., 1991, Du classement par niveau de prospérité à l'entretien de groupe homogène "focos group". Dakar, CRDI, Séminaire-atelier fév. 91,16 p. multig.

Khon Kaen University, 1985, Rapid rural appmisal proceedings of the 1985 International RRA Conference. Faculty of Agriculture, Khon Kaen University,Thailand, 357p.

MCCracken J.A., Pretty J.N., Conway G.R., 1988, Introduction au diagnostic rapide pour le développement en milieu rural. Londres, IIED, $69 \mathrm{p}$.

Olivier de Sardan J.P., 1991, A propos du populisme développementiste : idéologie, action, connaissance, note de lecture à propos de "Putting the last first" de R. Chambers, 21 p.multig.

ORSTOM/ UA 94 CNRS, 1986, L'exercice du développement. Paris, ORSTOM, Col. "colloques et séminaires", $355 \mathrm{p}$.

Simard G., 1989, Animer, planifier et évaluer l'action. La méthode du Focus group. Univ. de Laval (Canada), Mondia Editeur, 99 p.

Toulmin C., Chambers R., 1990, "Farmer-First" : pour un développement durable des zones arides d'Afrique. Londres, J McCracken, RRA Notes Network, 18 p.

\section{AUTEURS}

ABDOU SALAM FALL

Sociologue - Dakar

ANDRÉ LERICOLLAIS

Géographe - ORSTOM - Dakar 